\title{
Desafíos para la enfermería chilena en el contexto de pandemia según los lineamientos del Consejo Internacional de Enfermeras
}

Autor:

Erika Caballero Muñoz. Enfermera, Magister. Directora, Región América Latina y el Caribe, del Consejo Internacional de Enfermeras, Directora Escuela de Enfermería Universidad Católica Silva Henríquez

El Consejo Internacional de Enfermeras (CIE), es una federación de 130 asociaciones nacionales de enfermeras y más de 20 millones de enfermeras en todo el mundo. Desde su creación en 1899, su misión es promover el bienestar de las enfermeras, el avance de la profesión de enfermería y abogar por la salud en todas las políticas a nivel global (1).

El 2017 el CIE, la Organización Mundial de la Salud y Burdett Trust for Nursing lanzaron la campaña Nursing Now, "Enfermería Ahora", que buscaba relevar el rol e importancia de las enfermeras el sistema de salud, aumentar los puestos y mejorar condiciones de trabajo (2).

El año 2020 fue declarado el año de la enfermería, por el bicentenario del nacimiento de Florence Nigthingale (2); sin embargo, por la pandemia los ojos del mundo están puestos en nuestra profesión de una manera que no podríamos haber anticipado. En la región de América Latina el impacto del Covid 19 ha sido mayor por las determinantes sociales, que se caracterizan por un mayor nivel de pobreza, mayor hacinamiento, mayor migración, envejecimiento progresivo de la población, condiciones de trabajo más precarias que otras regiones, servicios de salud menos equitativos, con barreras de acceso y aspectos culturales diferentes (3).

El Covid-19 ha agravado la desigualdad de género en todo el mundo como las principales cuidadoras de niños, las mujeres se han visto afectadas por el cierre de escuelas y guarderías; con mayor probabilidad de estar en el trabajo a tiempo parcial, las 
mujeres han estado entre las primeras en ser despedidas; y las restricciones de quedarse en casa han resultado en una mayor violencia doméstica, como ha señalado el CIE (4).

La falta de equipo de protección individual ha sido un problema relevante, incluso en algunos países de la región, las propias enfermeras deben llevar sus equipos de protección. Se ha requerido también capacitación en manejo de pacientes en UCI de todos los profesionales de salud, especialmente en un sistema de salud sobrecargado y estresado. Otro problema asociado es el agotamiento profesional producto del estrés y cansancio extremo, frustración, impotencia, miedo, angustia y ansiedad. Existe también incertidumbre de los profesionales por la seguridad de sus propias familias; las(os) enfermeras(os) clínicos se han visto muy cercanos a la muerte de sus pacientes, muchas veces en condiciones poco dignas, lo que hace cuestionarse más aun, respecto a los aspectos éticos de la pandemia, y han sido el apoyo para miles de pacientes que están solos en el hospital. Las enfermeras(os) se han visto expuestos a violencia, con evidencias de discriminación y maltrato, por creer la población que son una fuente de contagio, lo que da cuenta de la falta de información (5).

La pandemia de COVID-19 ha empeorado gravemente la salud mental y el bienestar de las sociedades. Ha magnificado la crisis de salud mental existente y la necesidad de aumentar urgentemente la inversión en servicios de salud mental., es por esto por lo que el CIE ha instado a los gobiernos a actuar ahora, protegiendo al personal sanitario, su salud mental y a poniendo tolerancia cero a este tipo de actos (6).

Las 12 prioridades principales contra el COVID-19, que el CIE hace en su llamado a la acción de los gobiernos son (7):
- Priorizar urgentemente un acceso fácil a suficiente cantidad de equipos de protección individual (EPI) de alta calidad y apropiados para enfermeras y otros trabajadores sanitarios. Nuestro director general Howard Catton ha instado a los líderes del mundo a garantizar el suministro de equipos de protección individual.

- Garantizar que todas las enfermeras tienen formación apropiada en prevención y control de infecciones basada en la evidencia, así como la orientación y la capacitación específica más reciente sobre el COVID-19.

- Proteger la salud y el bienestar de las enfermeras(os) y otros trabajadores sanitarios.

- Implementar/intensificar rápidamente los sistemas de vigilancia integral para el seguimiento e investigación de la infección de trabajadores sanitarios.

- Garantizar la protección económica y la compensación de las enfermeras(os).

- Proporcionar una respuesta eficaz en materia de registro y regulación al aumentar rápidamente el personal de enfermería.

- Garantizar un despliegue seguro y eficaz del personal de enfermería en áreas de demanda y complejidad elevadas.

- Alentar, desarrollar y sostener nuevos modelos de cuidados e innovación.

- Demostrar apoyo público y reconocer el valor de las enfermeras(os) para la sociedad.

- Sacarle partido al liderazgo de la enfermería, haciéndolas participes de las mesas sociales de trabajo. 
- Desarrollar e implementar una estrategia de salud pública integral y coordinada contra el COVID-19 con la colaboración activa de las enfermeras(os).

- Aprender de la pandemia de COVID-19 al objeto de estar preparados para el futuro.

Muchas enfermeras(os) han muerto a nivel mundial. OMS ha declarado que al menos un $10 \%$ de los infectados por Covid son trabajadores de salud. No somos ni héroes, ni ángeles, nos hemos sacrificado, porque somos profesionales que amamos nuestra profesión y el rol que cumplimos con las personas, familias y comunidades. El Dr. Tedros Adhanom Ghebreyesus, Director General de la OMS, ha señalado el 17 de septiembre de 2020, con motivo del dia de la seguridad del paciente, que "Ningún país, hospital o clínica puede mantener seguros a sus pacientes a menos que mantenga seguros a sus trabajadores de salud (8). La Carta de seguridad de los trabajadores de la salud de la OMS es un paso para garantizar que los trabajadores de la salud tengan las condiciones de trabajo seguras, la capacitación, el salario y el respeto que merecen. En este sentido se ha pedido a los gobiernos y a quienes gestionan los servicios de salud a nivel local que tomen cinco medidas para proteger mejor a los trabajadores de la salud. Estos incluyen medidas para proteger a los trabajadores de la salud de la violencia; para mejorar su salud mental; protegerlos de peligros físicos y biológicos; promover programas nacionales para la seguridad de los trabajadores sanitarios y conectar las políticas de seguridad de los trabajadores sanitarios con las políticas de seguridad del paciente existentes (9).

En base a lo anterior y a la campaña Nursing Now, surgen muchos desafíos desde los lineamientos del ICN para la enfermería chilena 2020, tales como $(10,11)$ :
- Mayor inversión en educación, desarrollo profesional, regulación y condiciones de empleo de las enfermeras/os. Promover la enfermería avanzada, especialmente en la atención primaria, para resolver problemas de salud cada vez más complejos y generar evidencia para los responsables de las políticas y decisiones sobre donde la enfermeria puede tener el mayor impacto. Esto implica registro y regulación de la práctica y de la formación, contar con procesos de certificación de la profesión mediante un examen nacional y lograr la prescripción de medicamentos posterior a proceso de capacitación continua. Lo anterior lleva amarrado el diagnóstico de las carencias en formación práctica, la falta de profesorado con postgrados, así como limitación de infraestructuras y falta de lugares de prácticas.

- Eliminar el déficit de enfermería, para eso se deben tener datos reales del déficit. A nivel mundial se ha proyectado un déficit de $9 \mathrm{mi}$ llones de enfermeras para el 2030, se espera a nivel mundial que una de cada 6 enfermeras jubile en los próximos 10 años, por lo que se debe invertir en enfermería, mejorando las opciones de obtener empleo y retenerlo en el sistema de salud, con puestos de trabajo dignos, con protección económica y compensación para las enfermeras/os. Se debe asegurar entonces mejores condiciones laborales con un ambiente laboral seguro, respeto, sueldo adecuado, valor y reconocimiento. Hay que asegurar además que las cargas de trabajo sean acordes a sus responsabilidades; $y$ promover organizaciones de salud que aprenden a seguir mejorando continuamente.

- Promover una mayor influencia de las enfermeras en las políticas de salud, mediante 
la existencia de un Director(a) de enfermería o Jefa de enfermería de gobierno como parte del equipo directivo más importante en materia de salud. Cabe destacar que un $75 \%$ de los países, ya ha logrado esta posición. Esto impacta en la consecuente creaciones de planes nacionales de enfermería, políticas de dotación de enfermeras, e incluso de ser necesario podría requerirse una ley enfermera que regule la profesión, en cuanto a condiciones y horas de trabajo, protección, salario mínimo, funciones de avanzada, entre otras y constituya una manera de garantizar la salud de la población.

- Más enfermeras en posición de liderazgo, y promoción del liderazgo de los menores de 35 años, mediante la capacitación y apoyo de $\mathrm{CIE}$ en este aspecto. Establecimiento de una red de liderazgo en enfermería y red mundial de investigación.

- Alentar, desarrollar y sostener nuevos modelos de cuidados e innovación, con cuidado centrado en la persona, que tengan como brújula la humanización y compasión, modelos salutogénico, con enfoque preventivo promocional. Innovadoras modalidades de trabajo que incorporen la tecnología en el sector sanitario de manera segura, con estándares internacionales, con políticas clases y procesos de organización en materia de eventos adversos relacionados con la tecnológica.

- Demostrar apoyo público y reconocer el valor de las enfermeras/os en la sociedad, elevando el perfil e imagen profesional, tratar los temas de género y desigualdades.

- Otro desafío se relaciona con el costo de la atención y búsqueda de valor, dado el rol de gestión las profesionales de enfermería que permite crear cadenas de suministros sólidas y velar por el uso sostenibles de los recursos de salud.

- Promover el trabajo interdisciplinario, la formación interprofesional y colaborativa, teniendo alianzas fuera y dentro del sector sanitario. No se puede olvidar la migración, la movilidad y el cambio climático, como factores que siguen siendo un desafío que enfrenta la enfermería.

- Finalmente, un gran desafío es la unión de las asociaciones nacionales de enfermería con solidaridad y cohesión, para "Trabajar juntos", lema del CIE 2017-2021, facilitando el contar con organismos internacionales que trabajen junto en colaboración regional e internacional.

Sin duda alguna el Gobierno de Chile debe invertir en las enfermeras(os), en su formación, y optimizar las contribuciones de las enfermeras a las políticas sanitarias y a la prestación de servicios de salud más seguros y de calidad; las enfermeras(os) tenemos hoy una gran oportunidad de dar un paso firme, con evidencia científica, que permita llevar al mundo hacia la salud.

\section{Referencias bibliográficas}

1. International Council of Nurses (ICN). Quienes somos [internet]. ICN; 2020 [Actualizado 2020; acceso jun 2020]. Disponible en: https://www.icn. ch/es/quienes-somos\#main-content

2. International Council of Nurses (ICN). Nursing Now [internet]. ICN; 2019 [Actualizado 2020; acceso jun 2020]. Disponible en: https://www.icn. ch/es/que-hacemos/campanas/nursing-now

3. Comisión Económica para América Latina y el Caribe (CEPAL). Informe especial COVID-19. El 
desafío social en tiempos del COVID-19 [internet]. CEPAL; 2020 [Actualizado 12 may 2020; acceso jun 2020]. Disponible en: https://repositorio.cepal. org/bitstream/handle/11362/45527/5/S2000325_ es.pdf

4. All-Party Parliamentary Group on Global Health (APPG). Triple Impact. How developing nursing will improve health, promote gender equality, and support economic growth. [internet]. APPG; 2016 [Actualizado 17 oct 2016; acceso jun 2020]. Disponible en: https://www.who.int/hrh/comheeg/digital-APPG_triple-impact.pdf

5. International Council of Nurses (ICN). ACtualización del CIE sobre COVID 19: hay que minimizar los riesgos laborales de las enfermeras para que puedan seguir realizando su labor vital. [internet]. ICN; 2020 [Actualizado 30 mar 2020; acceso jun 2020]. Disponible en: https://www.icn.ch/ es/noticias/actualizacion-del-cie-sobre-la-covid19-hay-que-minimizar-los-riesgos-laborales-delas

6. International Council of Nurses (ICN). Position Statemen. Mental Health. [internet]. ICN; 2020 [Actualizado 2020; acceso jun 2020]. Disponible en: https://www.icn.ch/sites/default/files/inlinefiles/PS_A_Mental\%20Health_1.pdf

7. International Council of Nurses (ICN). Llamamiento del CIE a la acción. [internet]. ICN; 2020 [Actualizado 2020; acceso jun 2020]. Disponible en: https://www.icn.ch/system/files/documents/2020-04/ICN\%20briefing_COVID19_ Top_ priorities_SP.pdf

8. Organización Mundial de la Salud (OMS). OMS: Garantizar la seguridad de los trabajadores de la salud para preservar la de los pacientes. [internet]. OMS; 2020 [Actualizado 17 sep 2020; acceso sep 2020]. Disponible en: https://www.who.int/ es/news/item/17-09-2020-keep-health-workers- safe-to-keep-patients-safe-who

9. Organización Mundial de la Salud (OMS). La OMS y sus asociados hacen un llamamiento urgente para que se invierta en el personal de enfermería. [internet]. OMS; 2020 [Actualizado 7 abr 2020; acceso sep 2020]. Disponible en: https://www.who. int/es/news/item/07-04-2020-who-and-partnerscall-for-urgent-investment-in-nurses

10. Affara FA, Afuha'amango A, Al Darazi F, Aiken LH, Betker C, Buchan J, et al. Enfermería: Una voz para Liderar. Llevando al Mundo hacia la Salud. Ginebra (Suiza): Consejo Internacional de Enfermeras; 2020. 64p.

11. Stewart D, Burton E, White J, Salmon M, McClelland A. Enfermeras salud para todos enfermería, salud global y cobertura sanitaria universal día internacional de la enfermera. recursos y evidencias. Ginebra (Suiza): Consejo Internacional de Enfermeras; 2019. 60p. 